\title{
The interface is still the device
}

\author{
Oxide materials show an amazing variety of electronic and ionic phenomena. However, despite \\ considerable advances in understanding and utilizing these effects, experimental and theoretical \\ challenges still need to be addressed before the promised applications can be realized.
}

When Nobel laureate Herbert Kroemer coined the famous phrase that "the interface is the device", he referred to the astonishing success of devices based on thin semiconductor films for photonic and electronic applications that started more than 40 years ago. Now we are once more in the midst of a similar revolution, this time for oxide materials. Exhibiting a wide range of phenomena such as magnetism, superconductivity, ionic conduction and ferroelectricity, oxide materials are finding applications that include batteries, fuel cells, information storage and more. In particular, oxide interfaces, like their coventional semiconductor counterparts, offer the unique opportunity to control and enhance the effects by controlling the interaction between layers.

But where does this broad range of phenomena come from? For a start, the bonds between the ions in an oxide crystal tend to have a more polar character than in a conventional semiconductor such as silicon, which means that the electrons have stronger interactions with each other; they are said to be correlated. Moreover, the crystal structure of oxides is highly adaptable to changes in composition - many different chemical elements can be incorporated into the prototypical perovskite structure. This can not only be used for a very broad tuning of the carrier density in the crystal through doping, but it also increases the design flexibility in crystal composition. The latter is crucial to the development of improved materials such as ionic conductors for electrochemical applications, and moreover facilitates a wide range of different oxide compounds that can be deposited on top of each other in thin-film devices.

Progress - particularly in oxide thin-film growth technology - during the past decade has led to a large number of breakthroughs. For example, $\mathrm{LaAlO}_{3}$ and $\mathrm{SrTiO}_{3}$ are electrical insulators, but, grown on top of each other under the right conditions, they form a highly conducting layer at their interface (see image). This is just one of many cases where new functionality arises at oxide interfaces. A more detailed summary of these and other emergent phenomena is provided in the

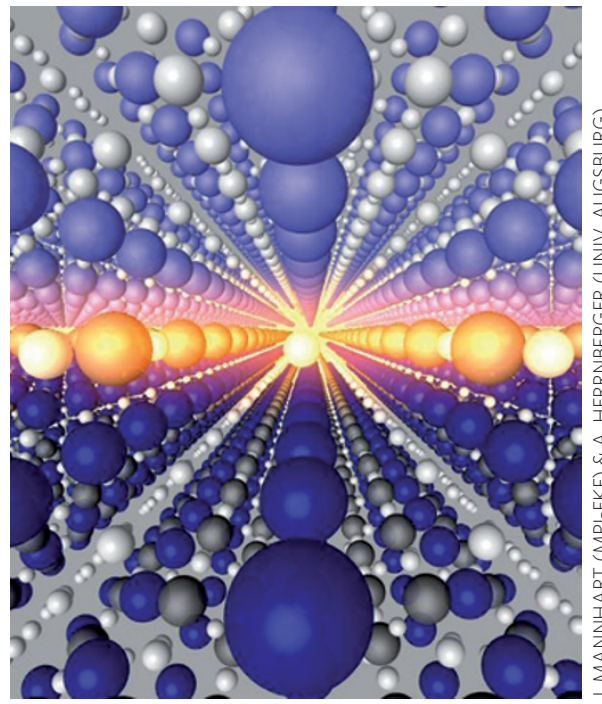

such growth can prove difficult owing to unfavourable energetics.

Researchers in the field therefore can't be complacent. Many of the applications touted in the past haven't materialized yet. Often the properties that make the oxides so interesting are difficult to realize, particularly at room temperature, or are too weak to be of practical use. Moreoever, some of these compounds contain expensive, rare elements that prohibit broader commercialization. At the fundamental level, some promising phenomena, such as topological insulating behaviour, have not yet been realized in oxides despite considerable efforts.

Nevertheless, progress has been made even under difficult circumstances. Oxide thin-films such as $\mathrm{LaAlO}_{3}$ and $\mathrm{SrTiO}_{3}$ have already been grown on silicon substrates, where avoiding the growth of undesired $\mathrm{SiO}_{2}$ is a considerable challenge. Although the full potential of emergent phenomena has not yet been realized for oxides grown on silicon, the marriage between conventional semiconductors and oxides clearly promises to combine semiconductor electronics with correlated electron systems.

It is clear that the advances made so far certainly warrant further research and strong funding for the field. Oxide thin-films have come a remarkably long way, and we are now beginning to understand how to design and control emergent phenomena in these materials systems. To appreciate just how far research in oxides has come and how exciting the prospects are for future research, Nature Materials, along with the Jülich-Aachen Research Alliance, is organizing a conference in June 2012 on 'Frontiers in Electronic Materials: Correlation Effects and Memristive Phenomena', where many of these advances will be discussed ${ }^{3}$. The programme will feature some of the most prolific researchers in the field, and will provide plenty of opportunity for discussion. We hope to see you there!

\footnotetext{
References

1. Hwang, H. Y. et al. Nature Mater. 11, 103-113 (2012).

2. Chakhalian, J., Millis, A. \& Rondinelli, J. Nature Mater. 11, 92-94 (2012).

3. http://www.nature.com/natureconferences/fem 2012
} 